\title{
Bicomplex Mittag-Leffler function and associated properties
}

\author{
Ritu Agarwala,*, Urvashi Purohit Sharmaa ${ }^{a}$, Ravi P. Agarwal ${ }^{b}$ \\ ${ }^{a}$ Department of Mathematics, Malaviya National Institute of Technology, Jaipur-302017, India. \\ ${ }^{b}$ Department of Mathematics, Texas A\&M University, Kingsville 700 University Blvd., Kingsville, USA.
}

\begin{abstract}
With the increasing importance of the Mittag-Leffler function in physical applications, these days many researchers are studying various generalizations and extensions of the Mittag-Leffler function. In this paper, efforts are made to define the bicomplex extension of the Mittag-Leffler function, and also its analyticity and region of convergence are discussed. Various properties of the bicomplex Mittag-Leffler function including integral representation, recurrence relations, duplication formula, and differential relations are established.
\end{abstract}

Keywords: Bicomplex numbers, exponential function, Gamma function, Mittag-Leffler function.

2020 MSC: 30G35, 33E12.

(C)2022 All rights reserved.

\section{Introduction}

Bicomplex numbers are being studied for quite a long time and a lot of work has been done in this area. Cockle $[9,10]$ introduced Tessarines between 1848 and 1850 following which Segre [42] introduced the bicomplex numbers. Many properties of the bicomplex numbers have been discovered. During the last few years researchers have aimed to study the different algebraic and geometric properties of bicomplex numbers and its applications (see, e.g., [11, 31, 37, 38, 40, 41]). In the recent developments, efforts have been done to extend the integral transforms [2, 3], holomorphic and meromorphic functions [11-13] a number of functions like Polygamma function [17], Hurwitz Zeta function [18], Gamma and Beta functions [19], Riemann Zeta function [37], bicomplex analysis and Hilbert space [24-29] in the bicomplex variable from their complex counterparts. Bicomplex numbers generalize both: the complex numbers and hyperbolic numbers. The theories which are based on both types of domains, complex and hyperbolic, may be unified by developing that theory for bicomplex functions. For example, the theory of relativistic physics and quantum physics [21]. Further, the theory of bicomplex functions can be applied to electromagnetism by treating the electric and magnetic fields together as a bicomplex field [4].

Recently, various generalizations and the extensions of the Mittag-Leffler function are defined by many authors $[1,5-8,15,16,20,22,23,43]$ which are useful in the study of fractional calculus. Mittag-Leffler

\footnotetext{
*Corresponding author

Email addresses: ragarwal.maths@mnit.ac.in (Ritu Agarwal), urvashius100@gmail.com (Urvashi Purohit Sharma), ravi.agarwal@tamuk. edu (Ravi P. Agarwal)
}

doi: 10.22436/jnsa.015.01.04

Received: 2021-03-24 Revised: 2021-05-02 Accepted: 2021-05-28 
function appears while studying the fractional form of various differential equations. For studying the bicomplex version of these fractional differential equations in bicomplex space, a bicomplex version of the Mittag-Leffler function would be required. In this paper, efforts are made to define the bicomplex version of the Mittag-Leffler function.

\subsection{Bicomplex numbers}

Segre [42] defined the set of bicomplex numbers as following.

Definition 1.1 (Bicomplex number). In terms of real components, the set of bicomplex numbers is defined as

$$
\mathbb{T}=\left\{\xi: \xi=x_{0}+i_{1} x_{1}+i_{2} x_{2}+j x_{3} \mid x_{0}, x_{1}, x_{2}, x_{3} \in \mathbb{R}\right\},
$$

and in terms of complex numbers it can be written as

$$
\mathbb{T}=\left\{\xi: \xi=z_{1}+i_{2} z_{2} \mid z_{1}, z_{2} \in \mathbb{C}\right\} .
$$

We shall use the notations: $x_{0}=\operatorname{Re}(\xi), x_{1}=\operatorname{Im}_{i_{1}}(\xi), x_{2}=\operatorname{Im}_{i_{2}}(\xi), x_{3}=\operatorname{Im}_{\mathfrak{j}}(\xi)$.

Segre discussed the presence of zero divisors which he called Nullifics. He noticed that the zerodivisors in bicomplex numbers constitute two ideals which he called the infinite set of nullifics. The set of all zero divisors is called null cone [39] defined as follows:

$$
\mathbb{N C}=\mathrm{O}_{2}=\left\{z_{1}+z_{2} i_{2} \mid z_{1}^{2}+z_{2}^{2}=0\right\} .
$$

Two non trivial idempotent zero divisors in $\mathbb{T}$, denoted by $e_{1}$ and $e_{2}$ are defined as follows [34]:

$$
e_{1}=\frac{1+i_{1} i_{2}}{2}=\frac{1+j}{2}, e_{2}=\frac{1-i_{1} i_{2}}{2}=\frac{1-j}{2}, e_{1} \cdot e_{2}=0, e_{1}+e_{2}=1, \text { and } e_{1}^{2}=e_{1}, e_{2}^{2}=e_{2} .
$$

Definition 1.2 (Idempotent representation). Every element $\xi \in \mathbb{T}$ has unique idempotent representation in terms of $e_{1}$ and $e_{2}$ defined by

$$
\xi=z_{1}+i_{2} z_{2}=\left(z_{1}-i_{1} z_{2}\right) e_{1}+\left(z_{1}+i_{1} z_{2}\right) e_{2}=\xi_{1} e_{1}+\xi_{2} e_{2},
$$

where $\xi_{1}=\left(z_{1}-i_{1} z_{2}\right)$ and $\xi_{2}=\left(z_{1}+i_{1} z_{2}\right)$.

Projection mappings $\mathrm{P}_{1}: \mathbb{T} \rightarrow \mathrm{T}_{1} \subseteq \mathbb{C}, \mathrm{P}_{2}: \mathbb{T} \rightarrow \mathrm{T}_{2} \subseteq \mathbb{C}$ for a bicomplex number $\xi=z_{1}+i_{2} z_{2}$ are defined as (see, e.g. [38]):

$$
\mathrm{P}_{1}(\xi)=\mathrm{P}_{1}\left(z_{1}+i_{2} z_{2}\right)=\mathrm{P}_{1}\left[\left(z_{1}-i_{1} z_{2}\right) e_{1}+\left(z_{1}+i_{1} z_{2}\right) e_{2}\right]=\left(z_{1}-i_{1} z_{2}\right) \in \mathrm{T}_{1}
$$

and

$$
P_{2}(\xi)=P_{2}\left(z_{1}+i_{2} z_{2}\right)=P_{2}\left[\left(z_{1}-i_{1} z_{2}\right) e_{1}+\left(z_{1}+i_{1} z_{2}\right) e_{2}\right]=\left(z_{1}+i_{1} z_{2}\right) \in T_{2}
$$

where

$$
\mathrm{T}_{1}=\left\{\xi_{1}=z_{1}-i_{1} z_{2} \mid z_{1}, z_{2} \in \mathbb{C}\right\} \text { and } \mathrm{T}_{2}=\left\{\xi_{2}=z_{1}+i_{1} z_{2} \mid z_{1}, z_{2} \in \mathbb{C}\right\} .
$$

Remark 1.3. The bicomplex space $\mathbb{T}$ can be written as the product

$$
\mathbb{T}=\mathrm{T}_{1} \times{ }_{\mathrm{e}} \mathrm{T}_{2}=\left\{\xi_{1} e_{1}+\xi_{2} e_{2}, \xi_{1} \in \mathrm{T}_{1}, \xi_{2} \in \mathrm{T}_{2}\right\} .
$$

Definition 1.4 (Bicomplex modulii). Let $\xi=z_{1}+i_{2} z_{2}=\xi_{1} e_{1}+\xi_{2} e_{2}=x_{0}+x_{1} i_{1}+x_{2} i_{2}+x_{3} j \in \mathbb{T}$ (see, e.g. $[32,35,38])$. The norm or the real modulus of $\xi$ is given by

$$
\|\xi\|=\sqrt{\left|z_{1}\right|^{2}+\left|z_{2}\right|^{2}}=\frac{1}{\sqrt{2}} \sqrt{\left|\xi_{1}\right|^{2}+\left|\xi_{2}\right|^{2}}=\sqrt{x_{0}^{2}+x_{1}^{2}+x_{2}^{2}+x_{3}^{2}}
$$


The $i_{1}$-modulus of $\xi$ is defined as

$$
|\xi|_{i_{1}}=\sqrt{z_{1}^{2}+z_{2}^{2}}
$$

The $i_{2}$-modulus of $\xi$ is defined as

$$
|\xi|_{i_{2}}=\sqrt{\left(\left|z_{1}\right|^{2}-\left|z_{2}\right|^{2}\right)+2 \operatorname{Re}\left(z_{1} \overline{z_{1}}\right) i_{2}}
$$

The $j$-modulus of $\xi$ is defined as

$$
|\xi|_{j}=\left|z_{1}-i_{1} z_{2}\right| e_{1}+\left|z_{1}+i_{1} z_{2}\right| e_{2} .
$$

The absolute value of $\xi$ is denoted by $|\xi|_{a b s}$, and is defined as

$$
|\xi|_{\text {abs }}=\sqrt{\left|z_{1}^{2}+z_{2}^{2}\right|}=\sqrt{\left|\left(z_{1}-i_{1} z_{2}\right)\left(z_{1}+i_{1} z_{2}\right)\right|}=\sqrt{\left|\xi_{1} \xi_{2}\right|}=\sqrt{\left|\xi_{1}\right|\left|\xi_{2}\right|} .
$$

Definition 1.5 (Argument). Let $\xi=z_{1}+i_{2} z_{2}=\xi_{1} e_{1}+\xi_{2} e_{2}=x_{0}+x_{1} i_{1}+x_{2} i_{2}+x_{3} j \in \mathbb{T}$, then hyperbolic argument (see, e.g. [30]) of $\xi$ is given by:

$$
\arg _{j}(\xi)=\arg \left(\xi_{1}\right) e_{1}+\arg \left(\xi_{1}\right) e_{2} .
$$

Let $\mathrm{U} \subseteq \mathbb{T}$ be an open set, and $\mathrm{g}: \mathrm{U} \rightarrow \mathbb{T}$ (see, e.g. [37, 41]). Also $g\left(z_{1}+i_{2} z_{2}\right)=g_{1}\left(z_{1}, z_{2}\right)+i_{2} g_{2}\left(z_{1}, z_{2}\right)$, then $g$ is $\mathbb{T}$-holomorphic iff $g_{1}$ and $g_{2}$ are holomorphic in $U$ and

$$
\frac{\partial g_{1}}{\partial z_{1}}=\frac{\partial g_{2}}{\partial z_{2}} \quad \text { and } \quad \frac{\partial g_{2}}{\partial z_{1}}=-\frac{\partial g_{1}}{\partial z_{2}} \text { on } \mathrm{u} \text {. }
$$

These equations are called the bicomplex Cauchy-Riemann equations (abbr. bicomplex CR-equations),

$$
g^{\prime}=\frac{\partial g_{1}}{\partial z_{1}}+i_{2} \frac{\partial g_{2}}{\partial z_{1}}
$$

In the following theorem, Riley [35] studied the convergence of bicomplex power series.

Theorem 1.6. Let

$$
\mathrm{N}(\xi)=\sqrt{\|\xi\|^{2}+\sqrt{\|\xi\|^{4}-|\xi|_{\mathrm{abs}}^{4}}}=\max \left(\left|\xi_{1}\right|,\left|\xi_{2}\right|\right),
$$

then $N(\xi)$ is a norm and if $\sum_{n=0}^{\infty} a_{n} \xi^{n}, a_{n}=b_{n} e_{1}+c_{n} e_{2}$ is a power series with component series $\sum_{n=0}^{\infty} b_{n} \xi_{1}^{n}$ and $\sum_{n=0}^{\infty} c_{n} \xi_{2}^{n}$, both having the same radius of convergence $R>0$, then $\sum_{n=0}^{\infty} a_{n} \xi^{n}$ converges for $N(\xi)<R$ and diverges for $\mathrm{N}(\xi)>\mathrm{R}$, where $\|\xi\|=\frac{1}{\sqrt{2}} \sqrt{\left|\xi_{1}\right|^{2}+\left|\xi_{2}\right|^{2}}$ and $|\xi|_{\mathrm{abs}}=\sqrt{\left|\xi_{1}\right|\left|\xi_{2}\right|}$.

In the following theorem, Ringleb [36] (see also, [35]) discussed the analyticity of a bicomplex function w.r.t. its idempotent complex component functions. This theorem plays a vital role while discussing the convergence of the bicomplex functions.

Theorem 1.7 (Decomposition theorem of Ringleb [36]). Let $\mathrm{f}(z)$ be analytic in a region $\mathrm{U} \subseteq \mathbb{T}$, and let $\mathrm{T}_{1} \subseteq \mathbb{C}$ and $\mathrm{T}_{2} \subseteq \mathbb{C}$ be the component regions of $\mathbb{T}$, in the $\xi_{1}$ and $\xi_{2}$ planes, respectively. Then there exists a unique pair of complex-valued analytic functions, $\mathrm{f}_{1}\left(\xi_{1}\right)$ and $\mathrm{f}_{2}\left(\xi_{2}\right)$, defined in $\mathrm{U}_{1} \subseteq \mathrm{T}_{1}$ and $\mathrm{U}_{2} \subseteq \mathrm{T}_{2}$, respectively, such that

$$
f(z)=f_{1}\left(\xi_{1}\right) e_{1}+f_{2}\left(\xi_{2}\right) e_{2}, \xi \in U .
$$

Conversely, if $\mathrm{f}_{1}\left(\xi_{1}\right)$ is any complex-valued analytic function in a region $\mathrm{T}_{1}$ and $\mathrm{f}_{2}\left(\xi_{2}\right)$ any complex-valued analytic function in a region $T_{2}$, then the bicomplex-valued function $f(z)$ defined by the equation (1.5) is an analytic function of the bicomplex variable $\xi$ in the product-region $\mathrm{U}=\mathrm{U}_{1} \times_{e} \mathrm{U}_{2}$. 
In the Theorem 1.8, Price [34] studied the integration in bicomplex domain w.r.to its idempotent representation. This theorem plays a basic role in the study of integrals of the bicomplex function.

Theorem 1.8. Let $\mathrm{U} \subseteq \mathbb{T}$. Let $\mathrm{C}_{1}, \mathrm{C}_{2}$ be two curves defined as

$$
\begin{aligned}
& C_{1}: z_{1}-i_{2} z_{2}=z_{1}(t)-i_{1} z_{2}(t)=\xi_{1}=\xi_{1}(t), a \leqslant t \leqslant b, \\
& C_{2}: z_{1}+i_{2} z_{2}=z_{1}(t)+i_{1} z_{2}(t)=\xi_{2}=\xi_{2}(t), a \leqslant t \leqslant b,
\end{aligned}
$$

which have continuous derivatives and whose traces are in $\mathrm{U}_{1} \subseteq \mathrm{T}_{1}, \mathrm{U}_{2} \subseteq \mathrm{T}_{2}$, respectively and let $\mathrm{C}$ be the curve with trace in $\mathrm{U}$ which is defined as

$$
C: \xi(t)=\xi_{1}(t) e_{1}+\xi_{2}(t) e_{2}, a \leqslant t \leqslant b .
$$

Then the integrals of $\mathrm{f}, \mathrm{f}_{1}, \mathrm{f}_{2}$ on the curves $\mathrm{C}, \mathrm{C}_{1}$, and $\mathrm{C}_{2}$ exist and

or

$$
\int_{C} f(\xi) d \xi=\int_{C_{1}} f_{1}\left(z_{1}-i_{1} z_{2}\right) d\left(z_{1}-i_{1} z_{2}\right) e_{1}+\int_{C_{2}} f_{1}\left(z_{1}+i_{1} z_{2}\right) d\left(z_{1}+i_{1} z_{2}\right) e_{2}
$$

$$
\int_{C} f(\xi) d \xi=\int_{C_{1}} f_{1}\left(\xi_{1}\right) d\left(\xi_{1}\right) e_{1}+\int_{C_{2}} f_{2}\left(\xi_{2}\right) d\left(\xi_{2}\right) e_{2} .
$$

We would require the definition of the bicomplex gamma function defined by Goyal et al. [19], in the Euler product form as follows:

$$
\frac{1}{\Gamma \xi}=\xi e^{\gamma \xi} \prod_{n=1}^{\infty}\left(\left(1+\frac{\xi}{n}\right) \exp \left(-\frac{\xi}{n}\right)\right), \xi \in \mathbb{T},
$$

provided that $z_{1} \neq \frac{-(m+l)}{2}$, and $z_{2} \neq i_{1}\left(\frac{l-m}{2}\right)$, where $m, l \in \mathbb{N} \cup\{0\}$. The Euler constant $\gamma(0 \leqslant \gamma \leqslant 1)$ is given by

$$
\gamma=\lim _{n \rightarrow \infty}\left(H_{n}-\log n\right), H_{n}=\sum_{k=1}^{n} \frac{1}{k} .
$$

Also, in idempotent form

$$
\Gamma \xi=\Gamma \xi_{1} e_{1}+\Gamma \xi_{2} e_{2}, \xi \in \mathbb{T},
$$

and in the integral form (see, e.g.[19]), for $p=p_{1} e_{1}+p_{2} e_{2}, p_{1}, p_{2} \in \mathbb{R}^{+}$,

$$
\Gamma \xi=\int_{H} e^{-p} p^{\xi-1} d p=\left(\int_{0}^{\infty} e^{-p_{1}} p_{1}^{\xi_{1}-1} d p_{1}\right) e_{1}+\left(\int_{0}^{\infty} e^{-p_{2}} p_{2}^{\xi_{2}-1} d p_{2}\right) e_{2}
$$

where $H=\left(\gamma_{1}, \gamma_{2}\right)$ and $\gamma_{1}: 0$ to $\infty, \gamma_{2}: 0$ to $\infty$.

\subsection{Mittag-Leffler function and its properties}

The Mittag-Leffler function (M-L function) comes intrinsically in the study of the fractional calculus. The importance of the M-L function in science and engineering is continuously increasing. It is very useful in the area of fractional modeling of real life problems.

The one parameter M-L function defined by Mittag-Leffler [33] is given by

$$
\mathbb{E}_{\alpha}(z)=\sum_{k=0}^{\infty} \frac{z^{k}}{\Gamma(\alpha k+1)}, \operatorname{Re}(\alpha)>0, \quad z \in \mathbb{C} .
$$

It comes from the Cauchy inequality for the Taylor coefficients and properties of the Gamma function (see, e.g.[16, p.18]) that $\exists$ a number $k \geqslant 0$ and a positive number $r(k)$ such that

$$
M_{\mathbb{E}_{\alpha}(r)}=\max _{|z|=r}\left|\mathbb{E}_{\alpha}(z)\right|<e^{r^{k}}, \forall r>r(k),
$$


hence $\mathbb{E}_{\alpha}(z)$ is an entire function of finite order. For each $\operatorname{Re}(\alpha)>0$, the order $\rho$ and type $\sigma$ of M-L function (1.8) is given by

$$
\rho=\lim \sup _{k \rightarrow \infty} \frac{k \log k}{\log \frac{1}{\left|a_{k}\right|}}=\frac{1}{\operatorname{Re}(\alpha)}
$$

and

$$
\sigma=\frac{1}{e \rho} \lim \sup _{k \rightarrow \infty}\left(k\left|a_{k}\right|^{\frac{\rho}{k}}\right)=1
$$

\section{Bicomplex one-parameter Mittag-Leffler function}

Here, we introduce the bicomplex one parameter Mittag-Leffler function defined by

$$
\mathbb{E}_{\alpha}(\xi)=\sum_{k=0}^{\infty} \frac{\xi^{k}}{\Gamma(\alpha k+1)}
$$

where $\xi, \alpha \in \mathbb{T}, \xi=z_{1}+i_{2} z_{2}$ and $\left|\operatorname{Im}_{j}(\alpha)\right|<\operatorname{Re}(\alpha)$.

The definition of bicomplex M-L function is well justified by the following theorem.

Theorem 2.1. Let $\xi, \alpha \in \mathbb{T}$, where $\xi=z_{1}+i_{2} z_{2}=\xi_{1} e_{1}+\xi_{2} e_{2}, \alpha=\alpha_{1} e_{1}+\alpha_{2} e_{2}=a_{0}+i_{1} a_{1}+i_{2} a_{2}+i_{1} i_{2} a_{3}$ with $\left|\operatorname{Im}_{\mathbf{j}}(\alpha)\right|<\operatorname{Re}(\alpha)$. Then

$$
\mathbb{E}_{\alpha}(\xi)=\sum_{k=0}^{\infty} \frac{\xi^{k}}{\Gamma(\alpha k+1)}
$$

Proof. Consider the function

$$
\mathbb{E}_{\alpha}(\xi)=\sum_{k=0}^{\infty} \frac{\xi^{k}}{\Gamma(\alpha k+1)}
$$

By using the idempotent representation

$$
\mathbb{E}_{\alpha}(\xi)=\sum_{k=0}^{\infty} \frac{\xi_{1}^{k}}{\Gamma\left(\alpha_{1} k+1\right)} e_{1}+\sum_{k=0}^{\infty} \frac{\xi_{2}^{k}}{\Gamma\left(\alpha_{2} k+1\right)} e_{2}=\mathbb{E}_{\alpha_{1}}\left(\xi_{1}\right) e_{1}+\mathbb{E}_{\alpha_{2}}\left(\xi_{2}\right) e_{2},
$$

where $\xi=\xi_{1} e_{1}+\xi_{2} e_{2}, \xi_{1} \in T_{1}, \xi_{2} \in T_{2}$ and $\alpha=\alpha_{1} e_{1}+\alpha_{2} e_{2}$. Now,

$$
\mathbb{E}_{\alpha_{1}}\left(\xi_{1}\right)=\sum_{k=0}^{\infty} \frac{\xi_{1}^{k}}{\Gamma\left(\alpha_{1} k+1\right)} \quad \text { and } \quad \mathbb{E}_{\alpha_{2}}\left(\xi_{2}\right)=\sum_{k=0}^{\infty} \frac{\xi_{2}^{k}}{\Gamma\left(\alpha_{2} k+1\right)}
$$

are complex M-L functions convergent for $\operatorname{Re}\left(\alpha_{1}\right), \operatorname{Re}\left(\alpha_{2}\right)>0, \xi_{1} \in T_{1} \subseteq \mathbb{C}, \xi_{2} \in T_{2} \subseteq \mathbb{C}$. Since $\mathbb{E}_{\alpha_{1}}\left(\xi_{1}\right)$ and $\mathbb{E}_{\alpha_{2}}\left(\xi_{2}\right)$ are convergent in $T_{1}, T_{2}$, respectively, by Ringleb decomposition theorem, (2.2) is also convergent in $\mathbb{T}$. Further, Let

$$
\alpha=a_{0}+i_{1} a_{1}+i_{2} a_{2}+i_{1} i_{2} a_{3}=\alpha_{1} e_{1}+\alpha_{2} e_{2},
$$

where $\alpha_{1}=\left(a_{0}+a_{3}\right)+i_{1}\left(a_{1}-a_{2}\right)$ and $\alpha_{2}=\left(a_{0}-a_{3}\right)+i_{1}\left(a_{1}+a_{2}\right)$.

Since $\operatorname{Re}\left(\alpha_{1}\right)>0$ and $\operatorname{Re}\left(\alpha_{2}\right)>0$,

$$
\begin{aligned}
& \Rightarrow a_{0}+a_{3}>0 \text { and } a_{0}-a_{3}>0, \\
& \Rightarrow\left|a_{3}\right|<a_{0}, \\
& \Rightarrow\left|\operatorname{Im}_{j}(\alpha)\right|<\operatorname{Re}(\alpha) .
\end{aligned}
$$

This completes the proof. 
By substituting the value of the bicomplex gamma function defined by equation (1.6) in the equation (2.3) we get the following representation for Mittag-Leffler function.

Theorem 2.2. Let $\xi, \alpha \in \mathbb{T}$ where $\xi=z_{1}+i_{2} z_{2}=\xi_{1} e_{1}+\xi_{2} e_{2}, \alpha=a_{0}+i_{1} a_{1}+i_{2} a_{2}+i_{1} i_{2} a_{3}=\alpha_{1} e_{1}+\alpha_{2} e_{2}$, with $\left|\operatorname{Im}_{j}(\alpha)\right|<\operatorname{Re}(\alpha)$, then

$$
\mathbb{E}_{\alpha}(\xi)=\sum_{k=0}^{\infty} \xi^{k}(\alpha k+1) e^{\gamma(\alpha k+1)} \prod_{n=1}^{\infty}\left(\left(1+\frac{(\alpha k+1)}{n}\right) \exp \left(-\frac{(\alpha k+1)}{n}\right)\right) .
$$

Remark 2.3. In integral form, with the help of (1.7) the bicomplex M-L function can be represented as

$$
\mathbb{E}_{\alpha}(\xi)=\sum_{k=0}^{\infty} \frac{\xi^{k}}{\int_{H} e^{-p} p^{\alpha k} d p}
$$

where $H=\left(\gamma_{1}, \gamma_{2}\right)$ as defined in (1.7).

For different values of the $\alpha$ we obtain various bicomplex functions as special cases. To mention, a few are:

1. for $\alpha=0$, we get bicomplex binomial function $\mathbb{E}_{0}(\xi)=\frac{1}{1-\xi},\|\xi\|<1$;

2. for $\alpha=1$, we get bicomplex exponential function $\mathbb{E}_{1}( \pm \xi)=e^{ \pm \xi}$;

3. for $\alpha=2$, we get bicomplex cosine function $\mathbb{E}_{2}\left(-\xi^{2}\right)=\cos \xi$;

4. for $\alpha=2$, we get bicomplex hyperbolic cosine function $\mathbb{E}_{2}\left(\xi^{2}\right)=\cosh \xi$;

5. for $\alpha=3$, we get the following function: $\mathbb{E}_{3}(\xi)=\frac{1}{2}\left(e^{\xi^{1 / 3}}+2 e^{-(1 / 2) \xi^{1 / 3}} \cos \left(\frac{\sqrt{3}}{2} \xi^{1 / 3}\right)\right)$;

6. for $\alpha=4$, we get following bicomplex relation: $\mathbb{E}_{4}(\xi)=\frac{1}{2}\left(\cos \left(\xi^{1 / 4}\right)+\cosh \left(\xi^{1 / 4}\right)\right)$.

Theorem 2.4. The bicomplex Mittag-Leffler function defined in equation (2.1) satisfies bicomplex Cauchy-Riemann equations.

Proof. By the result (2.3) we have,

$$
\begin{aligned}
\mathbb{E}_{\alpha}(\xi) & =\mathbb{E}_{\alpha_{1}}\left(\xi_{1}\right) e_{1}+\mathbb{E}_{\alpha_{2}}\left(\xi_{2}\right) e_{2} \\
& =\mathbb{E}_{\alpha_{1}}\left(z_{1}-i_{1} z_{2}\right) e_{1}+\mathbb{E}_{\alpha_{2}}\left(z_{1}+i_{1} z_{2}\right) e_{2} \\
& =\mathbb{E}_{\alpha_{1}}\left(z_{1}-i_{1} z_{2}\right)\left(\frac{1+i_{1} i_{2}}{2}\right)+\mathbb{E}_{\alpha_{2}}\left(z_{1}+i_{1} z_{2}\right)\left(\frac{1-i_{1} i_{2}}{2}\right) \\
& =\left(\frac{1}{2}\left(\mathbb{E}_{\alpha_{1}}\left(z_{1}-i_{1} z_{2}\right)+\mathbb{E}_{\alpha_{2}}\left(z_{1}+i_{1} z_{2}\right)\right)\right)+i_{2}\left(\frac{i_{1}}{2}\left(\mathbb{E}_{\alpha_{1}}\left(z_{1}-i_{1} z_{2}\right)-\mathbb{E}_{\alpha_{2}}\left(z_{1}+i_{1} z_{2}\right)\right)\right) \\
& =f_{1}\left(z_{1}, z_{2}\right)+i_{2} f_{2}\left(z_{1}, z_{2}\right)
\end{aligned}
$$

where $f_{1}\left(z_{1}, z_{2}\right)=\frac{1}{2}\left(\mathbb{E}_{\alpha_{1}}\left(z_{1}-i_{1} z_{2}\right)+\mathbb{E}_{\alpha_{2}}\left(z_{1}+i_{1} z_{2}\right)\right)$ and $f_{2}\left(z_{1}, z_{2}\right)=\frac{i_{1}}{2}\left(\mathbb{E}_{\alpha_{1}}\left(z_{1}-i_{1} z_{2}\right)-\mathbb{E}_{\alpha_{2}}\left(z_{1}+i_{1} z_{2}\right)\right)$. $\mathbb{E}_{\alpha_{i}}(i=1,2)$ are complex M-L functions. Now,

$$
\begin{array}{ll}
\frac{\partial f_{1}}{\partial z_{1}}=\frac{1}{2}\left(\mathbb{E}_{\alpha_{1}}^{\prime}\left(z_{1}-i_{1} z_{2}\right)+\mathbb{E}_{\alpha_{2}}^{\prime}\left(z_{1}+i_{1} z_{2}\right)\right), & \frac{\partial f_{1}}{\partial z_{2}}=\frac{-i_{1}}{2}\left(\mathbb{E}_{\alpha_{1}}^{\prime}\left(z_{1}-i_{1} z_{2}\right)-\mathbb{E}_{\alpha_{2}}^{\prime}\left(z_{1}+i_{1} z_{2}\right)\right), \\
\frac{\partial f_{2}}{\partial z_{1}}=\frac{i_{1}}{2}\left(\mathbb{E}_{\alpha_{1}}^{\prime}\left(z_{1}-i_{1} z_{2}\right)-\mathbb{E}_{\alpha_{2}}^{\prime}\left(z_{1}+i_{1} z_{2}\right)\right), & \frac{\partial f_{2}}{\partial z_{2}}=\frac{1}{2}\left(\mathbb{E}_{\alpha_{1}}^{\prime}\left(z_{1}-i_{1} z_{2}\right)+\mathbb{E}_{\alpha_{2}}^{\prime}\left(z_{1}+i_{1} z_{2}\right)\right) .
\end{array}
$$

From the above equations it can be observed that

$$
\frac{\partial f_{1}}{\partial z_{1}}=\frac{\partial f_{2}}{\partial z_{2}} \quad \text { and } \quad \frac{\partial f_{2}}{\partial z_{1}}=-\frac{\partial f_{1}}{\partial z_{2}} .
$$

Hence, bicomplex Cauchy-Riemann equations are satisfied by the bicomplex M-L function. 
Theorem 2.5. The bicomplex $M-L$ function $\mathbb{E}_{\alpha}(\xi),\left|\operatorname{Im}_{j}(\alpha)\right|<\operatorname{Re}(\alpha)$ is an entire function in the bicomplex domain.

Proof. Let $\sum_{n=0}^{\infty} a_{n} \xi^{n}$ represents a bicomplex power series, where $a_{n}, \xi, \in \mathbb{T}, a_{n}=b_{n} e_{1}+c_{n} e_{2}, \xi=$ $\xi_{1} e_{1}+\xi_{2} e_{2}$. Then by Ringleb decomposition theorem 1.7, the series

$$
\sum_{n=0}^{\infty} a_{n} \xi^{n}=\left(\sum_{n=0}^{\infty} b_{n} \xi_{1}^{n}\right) e_{1}+\left(\sum_{n=0}^{\infty} c_{n} \xi_{2}^{n}\right) e_{2}
$$

converges iff $\sum_{n=0}^{\infty} b_{n} \xi_{1}^{n}$ and $\sum_{n=0}^{\infty} c_{n} \xi_{2}^{n}$ converge in the complex domains (see, e.g. [35]). Now from equation (2.3), the Mittag -Leffler function can be decomposed as

$$
\mathbb{E}_{\alpha}(\xi)=\mathbb{E}_{\alpha_{1}}\left(\xi_{1}\right) e_{1}+\mathbb{E}_{\alpha_{2}}\left(\xi_{2}\right) e_{2}
$$

Since $\mathbb{E}_{\alpha_{1}}\left(\xi_{1}\right)=\sum_{k=0}^{\infty} \frac{\xi_{1}^{k}}{\Gamma\left(\alpha_{1} k+1\right)}, \operatorname{Re}\left(\alpha_{1}\right)>0$, and $\mathbb{E}_{\alpha_{2}}\left(\xi_{2}\right)=\sum_{k=0}^{\infty} \frac{\xi_{2}^{k}}{\Gamma\left(\alpha_{2} k+1\right)}, \quad \operatorname{Re}\left(\alpha_{2}\right)>0$ are complex Mittag Leffler functions with infinite radius of covergence (say R) [16, p.18], then

$$
\left|\xi_{1}\right|<R,\left|\xi_{2}\right|<R
$$

From equation (1.4),

$$
\mathrm{N}(\xi)=\sqrt{\|\xi\|^{2}+\sqrt{\|\xi\|^{4}-|\xi|_{a b s}^{4}}}=\max \left(\left|\xi_{1}\right|,\left|\xi_{2}\right|\right)<\mathrm{R} .
$$

As a consequence pf the Theorem 1.6, $\mathbb{E}_{\alpha}(\xi)$ converges in the bicomplex domain and has infinite radius of convergence [35]. Since complex M-L function is entire function in $\mathbb{C}$ the bicomplex M-L function is an entire function in $\mathbb{T}$ (Riley [35, p.141]).

Theorem 2.6 (Order and type). The bicomplex Mittag-Leffler function $\mathbb{E}_{\alpha}(\xi), \xi, \alpha \in \mathbb{T}$ is an entire function of finite order $\rho=\frac{a_{0}-a_{3} j}{\left(a_{0}^{2}-a_{3}^{2}\right)}$ and type $\sigma=1$.

Proof. From equation (2.3),

$$
\mathbb{E}_{\alpha}(\xi)=\mathbb{E}_{\alpha_{1}}\left(\xi_{1}\right) e_{1}+\mathbb{E}_{\alpha_{2}}\left(\xi_{2}\right) e_{2}
$$

Here $\mathbb{E}_{\alpha_{1}}\left(\xi_{1}\right)$ and $\mathbb{E}_{\alpha_{2}}\left(\xi_{2}\right)$ are the complex Mittag-Leffler functions for $\operatorname{Re}\left(\alpha_{1}\right)>0$ and $\operatorname{Re}\left(\alpha_{2}\right)>0$, respectively. Since $\mathbb{E}_{\alpha_{1}}\left(\xi_{1}\right), \mathbb{E}_{\alpha_{2}}\left(\xi_{2}\right)$ are entire functions, there exists numbers $k_{1}, k_{2} \geqslant 0$ and positive numbers $r_{1}\left(k_{1}\right), r_{2}\left(k_{2}\right)$, such that, from equation (1.9), we get

$$
M_{\mathbb{E}_{\alpha_{1}}\left(r_{1}\right)}=\max _{\left|\xi_{1}\right|=r_{1}}\left|\mathbb{E}_{\alpha_{1}}\left(\xi_{1}\right)\right|<e^{r_{1}^{k_{1}}}, \forall r_{1}>r_{1}\left(k_{2}\right)
$$

and

$$
M_{\mathbb{E}_{\alpha_{2}}\left(r_{2}\right)}=\max _{\left|\xi_{2}\right|=r_{1}}\left|\mathbb{E}_{\alpha_{2}}\left(\xi_{2}\right)\right|<e^{r_{2}^{k_{2}}}, \forall r_{2}>r_{2}\left(k_{2}\right) .
$$

Let $r=\max \left(r_{1}, r_{2}\right)$ and $k=\max \left(k_{1}, k_{2}\right)$, then

$$
M_{\mathbb{E}_{\alpha_{1}}\left(r_{1}\right)}=\max _{\left|\xi_{1}\right|=r_{1}}\left|\mathbb{E}_{\alpha_{1}}\left(\xi_{1}\right)\right|<e^{r_{1}^{k_{1}}} \leqslant e^{r^{k}},
$$

and

$$
\begin{aligned}
M_{\mathbb{E}_{\alpha_{2}}\left(\mathrm{r}_{2}\right)} & =\max _{\left|\xi_{2}\right|=r_{1}}\left|\mathbb{E}_{\alpha_{2}}\left(\xi_{2}\right)\right|<e^{r_{2}^{k_{2}}} \leqslant e^{r^{k}}, \\
M_{\mathbb{E}_{\alpha}(r)} & =\max _{|\xi|_{j}=r}\left|\mathbb{E}_{\alpha}(\xi)\right|_{j} \quad(J-m o d u l u s \text { of bicomplex number }) \\
& =\max _{\left|\xi_{1}\right|=r}\left|\mathbb{E}_{\alpha_{1}}\left(\xi_{1}\right)\right| e_{1}+\max _{\left|\xi_{2}\right|=r}\left|\mathbb{E}_{\alpha_{2}}\left(\xi_{2}\right)\right| e_{2} \leqslant e^{r^{k}} e_{1}+e^{r^{k}} e_{2}, \quad \forall r>r\left(k_{)}=e^{r^{k}} .\right.
\end{aligned}
$$


Hence $\mathbb{E}_{\alpha}(z)$ is an entire function of finite order.

For the bicomplex M-L function $\xi, \alpha \in \mathbb{T},\left|\operatorname{Im}_{j}(\alpha)\right|<\operatorname{Re}(\alpha)$, the order $\rho$ is given by

$$
\rho=\lim \sup _{k \rightarrow \infty} \frac{k \log k}{\log \Gamma(\alpha k+1)}=\left(\lim \sup _{k \rightarrow \infty} \frac{k \log k}{\log \Gamma\left(\alpha_{1} k+1\right)}\right) e_{1}+\left(\lim \sup _{k \rightarrow \infty} \frac{k \log k}{\log \Gamma\left(\alpha_{2} k+1\right)}\right) e_{2} .
$$

Now, from equation (1.10),

$$
\rho=\left(\frac{1}{\operatorname{Re}\left(\alpha_{1}\right)}\right) e_{1}+\left(\frac{1}{\operatorname{Re}\left(\alpha_{2}\right)}\right) e_{2}=\left(\frac{1}{a_{0}+a_{3}}\right) e_{1}+\left(\frac{1}{a_{0}-a_{3}}\right) e_{2}=\frac{a_{0}-a_{3} j}{\left(a_{0}^{2}-a_{3}^{2}\right)},
$$

where $a_{0}>\left|a_{3}\right| \Rightarrow a_{0}^{2}-a_{3}^{2} \neq 0$. The type $\sigma$ of the bicomplex M-L function $\mathbb{E}_{\alpha}(\xi)$ is given by

$$
\begin{aligned}
\sigma & =\frac{1}{e \rho} \lim \sup _{k \rightarrow \infty}\left(k\left|a_{k}\right|_{j}^{\frac{\rho}{k}}\right) \\
& =\frac{1}{e \rho} \lim \sup _{k \rightarrow \infty}\left(k\left|\frac{1}{\Gamma(\alpha k+1)}\right|_{j}^{\frac{\rho}{k}}\right) \\
& =\left(\frac{1}{e \rho} \lim \sup _{k \rightarrow \infty}\left(k\left|\frac{1}{\Gamma\left(\alpha_{1} k+1\right)}\right|^{\frac{\rho}{k}}\right)\right) e_{1}+\left(\frac{1}{e \rho} \lim \sup _{k \rightarrow \infty}\left(k\left|\frac{1}{\Gamma\left(\alpha_{2} k+1\right)}\right|^{\frac{\rho}{k}}\right)\right) e_{2} \\
& =1 . e_{1}+1 . e_{2}(\text { using equation (1.11)) } \\
& =1 .
\end{aligned}
$$

Remark 2.7. There are different modulus such as real, $i_{1}, i_{2}$, and $j$ modulus are defined for a bicomplex number (see, e.g. [38]). In this paper, $j$ modulus has been used for the calculation, since it provides expression in terms of idempotent components of the complex modulus.

\subsection{Properties of bicomplex Mittag-Leffler function}

Integral representation for the complex $\mathrm{M}-\mathrm{L}$ function $\mathbb{E}_{\alpha}(z)$ is given by (see, e.g.[14, p.209]):

$$
\int_{0}^{\infty} e^{-t} \mathbb{E}_{\alpha}\left(t^{\alpha} z\right) d t=\frac{1}{1-z}, z \in \mathbb{C}, \alpha \geqslant 0 .
$$

The above integral converges in unit circle and is bounded by the line $\operatorname{Re}\left(z^{1 / \alpha}\right)=1$.

Theorem 2.8 (Integral representation for bicomplex M-L function). Let $\xi \in \mathbb{T}$, where $\xi=z_{1}+i_{2} z_{2}=$ $\xi_{1} e_{1}+\xi_{2} e_{2}$ and $\alpha \geqslant 0,\|\xi\|<1$, then

$$
\int_{0}^{\infty} e^{-t} \mathbb{E}_{\alpha}\left(t^{\alpha} \xi\right) d t=\frac{1}{1-\xi}
$$

The above integral converges in the unit circle and is bounded by the plane $\operatorname{Re}\left(\xi^{1 / \alpha}\right)=1, \operatorname{Im}_{j}(\xi)=0$.

Proof. By the integral representation (2.4) and the result (2.3), for $\xi \in \mathbb{T}$, where $\xi=z_{1}+i_{2} z_{2}=\xi_{1} e_{1}+$ $\xi_{2} e_{2}, \quad \alpha \geqslant 0$ and $\left|\xi_{1}\right|<1,\left|\xi_{2}\right|<1$,

$$
\begin{aligned}
\int_{0}^{\infty} e^{-t} \mathbb{E}_{\alpha}\left(t^{\alpha} \xi\right) d t & =\left(\int_{0}^{\infty} e^{-t} \mathbb{E}_{\alpha}\left(t^{\alpha} \xi_{1}\right) d t\right) e_{1}+\left(\int_{0}^{\infty} e^{-t} \mathbb{E}_{\alpha}\left(t^{\alpha} \xi_{2}\right) d t\right) e_{2} \\
& =\left(\frac{1}{1-\xi_{1}}\right) e_{1}+\left(\frac{1}{1-\xi_{2}}\right) e_{2} \\
& =\frac{1}{1-\left(\xi_{1} e_{1}+\xi_{2} e_{2}\right)} \\
& =\frac{1}{1-\xi} .
\end{aligned}
$$


In terms of the real components, $\xi=x_{0}+i_{1} x_{1}+i_{2} x_{2}+j x_{3}=\xi_{1} e_{1}+\xi_{2} e_{2}$. Therefore, $\xi_{1}=\left(x_{0}+x_{3}\right)+i_{1}\left(x_{1}-\right.$ $\left.x_{2}\right), \xi_{2}=\left(x_{0}-x_{3}\right)+i_{1}\left(x_{1}+x_{2}\right)$. Since,

$$
\begin{aligned}
\left|\xi_{1}\right|<1 \text { and }\left|\xi_{2}\right|<1 & \Rightarrow \sqrt{\left(x_{0}+x_{3}\right)^{2}+\left(x_{1}-x_{2}\right)^{2}}<1 \text { and } \sqrt{\left(x_{0}-x_{3}\right)^{2}+\left(x_{1}+x_{2}\right)^{2}}<1 \\
& \Rightarrow \sqrt{x_{0}^{2}+x_{1}^{2}+x_{2}^{2}+x_{3}^{2}+2 x_{0} x_{3}-2 x_{1} x_{2}}<1 \text { and } \sqrt{x_{0}^{2}+x_{1}^{2}+x_{2}^{2}+x_{3}^{2}-2 x_{0} x_{3}+2 x_{1} x_{2}}<1 \\
& \Rightarrow \sqrt{x_{0}^{2}+x_{1}^{2}+x_{2}^{2}+x_{2}^{2}}<1 \\
& \Rightarrow\|\xi\|<1 .
\end{aligned}
$$

Also,

$$
\begin{aligned}
\operatorname{Re} \xi_{1}^{1 / \alpha}=1, \operatorname{Re} \xi_{2}^{1 / \alpha}=1 & \Rightarrow\left(x_{0}+x_{3}\right)^{\frac{1}{\alpha}}=1,\left(x_{0}-x_{3}\right)^{\frac{1}{\alpha}}=1 \\
& \Rightarrow\left(x_{0}+x_{3}\right)=1,\left(x_{0}-x_{3}\right)=1 \\
& \Rightarrow x_{0}=1, x_{3}=0 \\
& \Rightarrow \operatorname{Re} \xi=1, \operatorname{Im}_{j} \xi=0 \\
& \Rightarrow \operatorname{Re} \xi^{1 / \alpha}=1, \operatorname{Im}_{j} \xi=0 .
\end{aligned}
$$

The complex Mittag-Leffler function has following integral representation (see, e.g. [14])

$$
\mathbb{E}_{\alpha}(z)=\frac{1}{2 \pi i} \int_{\Omega} \frac{t^{\alpha-1} e^{t}}{t^{\alpha}-z} d t, \alpha>0, z \in \mathbb{C},
$$

where the path of integration $\Omega$ is a loop starting and ending at $-\infty$ and encircling the circular disk $|t| \leqslant|z|^{1 / \alpha}$ in the positive sense, $|\arg t|<\pi$ on $\Omega$.

Theorem 2.9. Let $\xi, \omega \in \mathbb{T}$, where $\xi=z_{1}+i_{2} z_{2}=\xi_{1} e_{1}+\xi_{2} e_{2}, \omega=\omega_{1} e_{1}+\omega_{2} e_{2}$, then bicomplex MittagLeffler function has following integral representation

$$
\mathbb{E}_{\alpha}(\xi)=\frac{1}{2 \pi i_{1}} \int_{H} \frac{\left.\omega^{\alpha-1} e^{(} \omega\right)}{\omega^{\alpha}-\xi} \mathrm{d} \omega, \alpha>0,
$$

where the path of integration $\mathrm{H}=\left(\Omega_{1}, \Omega_{2}\right)$ and $\Omega_{1}, \Omega_{2}$ are loops starting and ending at $-\infty$ and encircling the circular disks $\left|\omega_{1}\right| \leqslant\left|\xi_{1}\right|^{1 / \alpha},\left|\omega_{2}\right| \leqslant\left|\xi_{2}\right|^{1 / \alpha}$, respectively, in the positive sense, $\left|\arg \omega_{1}\right|<\pi$ on $\Omega_{1}$ and $\left|\arg \omega_{2}\right|<\pi$ on $\Omega_{2}$ equivalently, $\left|\arg _{j} \omega\right|_{j}<\pi$.

Proof. By the integral representation (2.6), result (2.3), and the Theorem 1.8, we have for $\xi, \omega \in \mathbb{T}$,

$$
\begin{aligned}
\mathrm{E}_{\alpha}(\xi) & =\mathrm{E}_{\alpha}\left(\xi_{1}\right) e_{1}+\mathrm{E}_{\alpha}\left(\xi_{2}\right) e_{2} \\
& =\frac{1}{2 \pi i_{1}} \int_{\Omega_{1}} \frac{\omega_{1}^{\alpha-1} e^{\omega_{1}}}{\omega_{1}^{\alpha}-\xi_{1}} \mathrm{~d} \omega_{1} e_{1}+\frac{1}{2 \pi i_{1}} \int_{\Omega_{2}} \frac{\omega_{2}^{\alpha-1} e^{\omega_{2}}}{\omega_{2}^{\alpha}-\xi_{2}} \mathrm{~d} \omega_{2} e_{2} \\
& =\frac{1}{2 \pi i_{1}} \int_{\left(\Omega_{1}, \Omega_{2}\right)} \frac{\left(\omega_{1} e_{1}+\omega_{2} e_{2}\right)^{\alpha-1} e^{\left(\omega_{1} e_{1}+\omega_{2} e_{2}\right)}}{\left(\omega_{1} e_{1}+\omega_{2} e_{2}\right)^{\alpha}-\left(\xi_{1} e_{1}+\xi_{2} e_{2}\right)} d\left(\omega_{1} e_{1}+\omega_{2} e_{2}\right) \\
& =\frac{1}{2 \pi i_{1}} \int_{H} \frac{\omega^{\alpha-1} e^{\omega}}{\omega^{\alpha}-\xi} d \omega .
\end{aligned}
$$

The path of integration is $H=\left(\Omega_{1}, \Omega_{2}\right)$, where $\Omega_{1}, \Omega_{2}$ are loops starting and ending at $-\infty$ and encircling the circular disks $\left|\omega_{1}\right| \leqslant\left|\xi_{1}\right|^{1 / \alpha},\left|\omega_{2}\right| \leqslant\left|\xi_{2}\right|^{1 / \alpha}$, respectively, in the positive sense. Further, since

$$
\left|\arg \omega_{1}\right|<\pi \text { and }\left|\arg \omega_{2}\right|<\pi,
$$


from the equations (1.2) and (1.3) we have

$$
\arg _{j} \omega=\left(\arg \omega_{1}\right) e_{1}+\left(\arg \omega_{2}\right) e_{2} \quad \Rightarrow \quad\left|\arg _{j} \omega\right|_{j}=\left|\arg \omega_{1}\right| e_{1}+\left|\arg \omega_{2}\right| e_{2}<\pi e_{1}+\pi e_{2}=\pi .
$$

Recurrence relation for the complex M-L function $\mathbb{E}_{\alpha}(z)$ is given by the following relation, where $p, q$ are the relatively prime natural numbers (see, e.g. [16, p.21])

$$
\mathbb{E}_{\mathfrak{p} / \mathrm{q}}(z)=\frac{1}{q} \sum_{l=0}^{q-1} \mathbb{E}_{1 / \mathfrak{p}}\left(z^{1 / \mathrm{q}} e^{\frac{2 \pi l i_{1}}{q}}\right) .
$$

Theorem 2.10 (Recurrence relation for bicomplex M-L function). Let $\xi \in \mathbb{T}$, where $\xi=z_{1}+i_{2} z_{2}$ and $\mathrm{p}, \mathrm{q} \in \mathbb{N}$ are relatively prime. Then the bicomplex Mittag-Leffler function satisfies

$$
\mathbb{E}_{\mathfrak{p} / \mathrm{q}}(\xi)=\frac{1}{q} \sum_{\mathrm{l}=0}^{\mathrm{q}-1} \mathbb{E}_{1 / \mathrm{p}}\left(\xi^{1 / \mathrm{q}} e^{\frac{2 \pi \mathrm{l}_{1}}{\mathrm{q}}}\right) .
$$

Proof. By the recurrence relation (2.7) and the result (2.3) we have for $\xi=z_{1}+i_{2} z_{2}=\xi_{1} e_{1}+\xi_{2} e_{2}=$ $\left(z_{1}-i_{1} z_{2}\right) e_{1}+\left(z_{1}+i_{1} z_{2}\right) e_{2}$

$$
\begin{aligned}
& \mathbb{E}_{\mathbf{p} / \mathbf{q}}(\xi)=\mathbb{E}_{\mathbf{p} / \mathbf{q}}\left(\xi_{1}\right) e_{1}+\mathbb{E}_{\mathbf{p} / \mathbf{q}}\left(\xi_{2}\right) e_{2}, q \in \mathbb{N} \\
& =\mathbb{E}_{\mathrm{p} / \mathrm{q}}\left(z_{1}-i_{1} z_{2}\right) e_{1}+\mathbb{E}_{\mathrm{p} / \mathrm{q}}\left(z_{1}+i_{1} z_{2}\right) e_{2} \\
& =\left(\frac{1}{q} \sum_{l=0}^{q-1} \mathbb{E}_{1 / p}\left(\left(z_{1}-i_{1} z_{2}\right)^{1 / q} e^{\frac{2 \pi l i_{1}}{q}}\right)\right) e_{1}+\left(\frac{1}{q} \sum_{l=0}^{q-1} \mathbb{E}_{1 / p}\left(\left(z_{1}+i_{1} z_{2}\right)^{1 / q} e^{\frac{2 \pi l i_{1}}{q}}\right)\right) e_{2} \\
& =\frac{1}{q} \sum_{l=0}^{q-1} \mathbb{E}_{1 / p}\left(\xi^{1 / q} e^{\frac{2 \pi l i_{1}}{q}}\right) \text {. }
\end{aligned}
$$

Duplication formula for the complex M-L function $\mathbb{E}_{\alpha}(z)$ is defined as (see, e.g. [16, p.53]):

$$
\mathbb{E}_{2 \alpha}\left(z^{2}\right)=\frac{1}{2}\left(\mathbb{E}_{\alpha}(z)+\mathbb{E}_{\alpha}(-z)\right), \operatorname{Re}(\alpha)>0 .
$$

Theorem 2.11 (Duplication formula for bicomplex M-L function). Let $\xi, \alpha \in \mathbb{T}$, where $\xi=z_{1}+i_{2} z_{2},\left|\operatorname{Im}_{j}(\alpha)\right|<$ $\operatorname{Re}(\alpha)$, then

$$
\mathbb{E}_{2 \alpha}\left(\xi^{2}\right)=\frac{1}{2}\left(\mathbb{E}_{\alpha}(\xi)+\mathbb{E}_{\alpha}(-\xi)\right) .
$$

Proof. We have for $\xi, \alpha \in \mathbb{T}$, where $\xi=z_{1}+i_{2} z_{2}=\xi_{1} e_{1}+\xi_{2} e_{2}=\left(z_{1}-i_{1} z_{2}\right) e_{1}+\left(z_{1}+i_{1} z_{2}\right) e_{2}$ and $\alpha=$ $\alpha_{1} e_{1}+\alpha_{2} e_{2},\left|\operatorname{Im}_{j}(\alpha)\right|<\operatorname{Re}(\alpha)$,

$$
\begin{aligned}
\frac{1}{2}\left(\mathbb{E}_{\alpha}(\xi)+\mathbb{E}_{\alpha}(-\xi)\right) & =\frac{1}{2}\left(\sum_{k=0}^{\infty} \frac{\xi^{k}}{\Gamma(\alpha \mathrm{k}+1)}+\sum_{\mathrm{k}=0}^{\infty} \frac{(-\xi)^{\mathrm{k}}}{\Gamma(\alpha \mathrm{k}+1)}\right) \\
& =\frac{1}{2} \sum_{\mathrm{k}=0}^{\infty} \frac{\left(\xi^{\mathrm{k}}+(-\xi)^{\mathrm{k}}\right)}{\Gamma(\alpha \mathrm{k}+1)} \\
& =\frac{1}{2} \sum_{\mathrm{k}=0}^{\infty} \frac{\left(\xi^{\mathrm{k}}\left(1+(-1)^{\mathrm{k}}\right)\right)}{\Gamma(\alpha \mathrm{k}+1)}
\end{aligned}
$$




$$
\begin{aligned}
& =\frac{1}{2}\left(2+2 \frac{\xi^{2}}{\Gamma(2 \alpha+1)}+2 \frac{\xi^{4}}{\Gamma(4 \alpha+1)}+2 \frac{\xi^{6}}{\Gamma(6 \alpha+1)}+\cdots\right) \\
& =\left(1+\frac{\left(\xi^{2}\right)^{1}}{\Gamma(1(2 \alpha)+1)}+\frac{\left(\xi^{2}\right)^{2}}{\Gamma(2(2 \alpha)+1)}+\frac{\left(\xi^{2}\right)^{3}}{\Gamma(3(2 \alpha)+1)}+\cdots\right) \\
& =\sum_{k=0}^{\infty} \frac{\left(\xi^{2}\right)^{k}}{\Gamma(2 \alpha k+1)} \\
& =\mathbb{E}_{2 \alpha}\left(\xi^{2}\right) .
\end{aligned}
$$

Differential relations for the complex M-L function $\mathbb{E}_{\alpha}(z)$ are defined by the following relations, where $p, q \in \mathbb{N}$ are relatively prime (see, e.g. [16, p.22]):

$$
\left(\frac{d}{d z}\right)^{p} \mathbb{E}_{\mathfrak{p}}\left(z^{p}\right)=\mathbb{E}_{\mathfrak{p}}\left(z^{p}\right), \quad \frac{d^{p}}{d z^{p}} \mathbb{E}_{\mathfrak{p} / \mathfrak{q}}\left(z^{p / q}\right)=\mathbb{E}_{\mathfrak{p} / \mathfrak{q}}\left(z^{p / q}\right)+\sum_{k=1}^{q-1} \frac{z^{-k p / q}}{\Gamma(1-k p / q)} .
$$

Theorem 2.12 (Differential relations for the bicomplex M-L function). Let $\xi \in \mathbb{T}$, where $\xi=z_{1}+i_{2} z_{2}$, then for $p, q$, relatively prime natural numbers,

(i) $\left(\frac{d}{d \xi}\right)^{p} \mathbb{E}_{p}\left(\xi^{p}\right)=\mathbb{E}_{p}\left(\xi^{p}\right) ;$

(ii) $\frac{d^{p}}{d \xi^{p}} \mathbb{E}_{p / q}\left(\xi^{p / q}\right)=\mathbb{E}_{p / q}\left(\xi^{p / q}\right)+\sum_{k=1}^{q-1} \frac{\xi^{-k p / q}}{\Gamma(1-k p / q)}$.

Proof.

(i)

$$
\begin{aligned}
& \left(\frac{d}{d \xi}\right)^{p} \mathbb{E}_{p}\left(\xi^{p}\right)=\left(\frac{d}{d \xi}\right)^{p} \sum_{k=0}^{\infty} \frac{\xi^{p k}}{\Gamma(p k+1)} \text { (From definition (2.1)) } \\
& =\sum_{k=1}^{\infty} \frac{\xi^{p k-p}}{\Gamma(p k-p+1)}=\sum_{k=0}^{\infty} \frac{\xi^{p k}}{\Gamma(p k+1)}(\text { Replacing } k \rightarrow k+1)=\mathbb{E}_{p}\left(\xi^{p}\right) \text {. }
\end{aligned}
$$

(ii) Again,

$$
\frac{d^{p}}{d \xi^{p}} \mathbb{E}_{p / q}\left(\xi^{p / q}\right)=\frac{d^{p}}{d \xi^{p}} \sum_{k=0}^{\infty} \frac{\xi^{\frac{k p}{q}}}{\Gamma\left(\frac{k p}{q}+1\right)}=\sum_{k=0}^{\infty} \frac{\xi^{\left(\frac{k}{q}-1\right) p}}{\Gamma\left(\frac{k p}{q}-p+1\right)}=\sum_{k=0}^{q-1} \frac{\xi^{\left(\frac{k p}{q}-p\right)}}{\Gamma\left(\frac{k p}{q}-p+1\right)}+\sum_{k=0}^{\infty} \frac{\xi^{\left(\frac{k p}{q}\right)}}{\Gamma\left(\frac{k p}{q}+1\right)} .
$$

The above equation can further be written as

$$
\frac{d^{p}}{d \xi^{p}} \mathbb{E}_{p / q}\left(\xi^{p / q}\right)=\sum_{k=1}^{q-1} \frac{\xi^{-k p / q}}{\Gamma(1-k p / q)}+\mathbb{E}_{p / q}\left(\xi^{p / q}\right)
$$

Theorem 2.13. The function $\mathbb{E}_{n}\left(\xi^{n}\right), \xi \in \mathbb{T}, n=1,2,3, \ldots$, satisfies the $\mathrm{n}^{\text {th }}$ order ordinary differential equation

$$
\frac{d^{n}}{d \xi^{n}}\left(\mathbb{E}_{n}\left(\xi^{n}\right)\right)=\mathbb{E}_{n}\left(\xi^{n}\right) .
$$


Proof. For $\alpha>0$, By replacing $\xi$ by $\xi^{\alpha}$ in the equation (2.1), we get

$$
\mathbb{E}_{\alpha}\left(\xi^{\alpha}\right)=\sum_{k=0}^{\infty} \frac{\xi^{\alpha k}}{\Gamma(\alpha k+1)}=1+\frac{\xi^{\alpha}}{\Gamma(\alpha+1)}+\frac{\xi^{2 \alpha}}{\Gamma(2 \alpha+1)}+\frac{\xi^{3 \alpha}}{\Gamma(3 \alpha+1)}+\cdots
$$

By taking derivative of order $\alpha$ on both sides of the equation (2.8), we get,

$$
\begin{aligned}
\mathrm{D}^{\alpha}\left(\mathbb{E}_{\alpha}\left(\xi^{\alpha}\right)\right) & =\mathrm{D}^{\alpha}\left(1+\frac{\xi^{\alpha}}{\Gamma(\alpha+1)}+\frac{\xi^{2 \alpha}}{\Gamma(2 \alpha+1)}+\frac{\xi^{3 \alpha}}{\Gamma(3 \alpha+1)}+\cdots\right) \\
& =\frac{\Gamma(1)}{\Gamma(1-\alpha)} \xi^{-\alpha}+\frac{\Gamma(\alpha+1)}{\Gamma(1)} \frac{1}{\Gamma(\alpha+1)}+\frac{\Gamma(2 \alpha+1)}{\Gamma(\alpha+1)} \frac{\xi^{\alpha}}{\Gamma(2 \alpha+1)}+\frac{\Gamma(3 \alpha+1)}{\Gamma(2 \alpha+1)} \frac{\xi^{2 \alpha}}{\Gamma(3 \alpha+1)}+\cdots \\
& =\frac{\Gamma(1)}{\Gamma(1-\alpha)} \xi^{-\alpha}+1+\frac{\xi^{\alpha}}{\Gamma(\alpha+1)}+\frac{\xi^{2 \alpha}}{\Gamma(2 \alpha+1)}+\cdots
\end{aligned}
$$

Since $\frac{1}{\Gamma(1-\alpha)}=0$ for $\alpha=n \in \mathbb{N}$, we get from equation (2.9),

$$
\mathrm{D}^{\mathrm{n}}\left(\mathbb{E}_{\mathrm{n}}\left(\xi^{\mathrm{n}}\right)\right)=1+\frac{\xi^{\mathrm{n}}}{\Gamma(\mathrm{n}+1)}+\frac{\xi^{2 \mathrm{n}}}{\Gamma(2 \mathrm{n}+1)}+\cdots=\sum_{k=0}^{\infty} \frac{\xi^{n k}}{\Gamma(\mathrm{nk}+1)}=\mathbb{E}_{n}\left(\xi^{n}\right) .
$$

\section{Conclusion}

In this paper, one parameter M-L function and its properties in bicomplex space have been defined from its complex counterpart. Various properties and special cases along with recurrence relations, duplication formula, integral representation, differential relation are also derived. We intend to extend the concepts of the fractional calculus in bicomplex space using the M-L function. Since bicomplex space provides a more generalized approach towards the large class of functions appearing in signal theory, electromagnetism, and quantum theory.

\section{Acknowledgment}

Authors are thankful to the referees for their constructive comments, which helped us to improve the manuscript.

\section{References}

[1] P. Agarwal, M. Chand, D. Baleanu, D. O'Regan, S. Jain, On the solutions of certain fractional kinetic equations involving k-Mittag-Leffler function, Adv. Difference Equ., 2018 (2018), 13 pages. 1

[2] R. Agarwal, M. P. Goswami, R. P. Agarwal, Mellin transform in bicomplex space and its application, Stud. Univ. Babeş-Bolyai Math., 62 (2017), 217-232. 1

[3] R. Agarwal, M. P. Goswami, R. P. Agarwal, Sumudu Transform In Bicomplex Space And Its Application, Ann. Appl. Math., 33 (2017), 239-253. 1

[4] R. Agarwal, M. P. Goswami, R. P. Agarwal, K. K. Venkataratnam, D. Baleanu, Solution of Maxwell's Wave Equations in Bicomplex Space, Romanian J. Phys., 62 (2017), 1-11. 1

[5] P. Agarwal, J. J. Nieto, some fractional integral formulas for the Mittag-Leffer type function with four parameters, Open Math., 13 (2015), 537-546. 1

[6] P. Agarwal, S. V. Rogosin, J. J. Trujillo, Certain fractional integral operators and the generalized multi-index Mittag-Leffler functions, Proc. Indian Acad. Sci. Math. Sci., 125 (2015), 291-306.

[7] M. Andrić, G. Farid, J. Pečarić, A further extension of Mittag-Leffler function, Fract. Calc. Appl. Anal., 21 (2018), 1377-1395.

[8] M. Arshad, J. Choi, S. Mubeen, K. S. Nisar, G. Rahman, A new extension of the Mittag-Leffler function, Commun. Korean Math. Soc., 33 (2018), 549-560. 1 
[9] J. Cockle, On a new Imaginary in Algebra, London, Edinburgh Dublin Philos. Mag. J. Sci., 34 (1849), 37-47. 1

[10] J. Cockle, T. S. Davis On certain Functions resembling Quaternions, and on a new Imaginary in Algebra, London, Edinburgh Dublin Philos. Mag. J. Sci., 33 (1848), 435-439. 1

[11] K. S. Charak, D. Rochon, On Factorization of Bicomplex Meromorphic Functions, In: Hypercomplex Analysis, Birkhäuser Verlag, Basel, (2009), 55-68. 1

[12] K. S. Charak, D. Rochon, N. Sharma, Normal Families of Bicomplex Holomorphic Functions, Fractals, 17 (2009), $257-$ 268.

[13] K. S. Charak, D. Rochon, N. Sharma, Normal Families of Bicomplex Meromorphic Functions, Ann. Polon. Math., 103 (2012), 303-317. 1

[14] A. Erdélyi, W. Magnus, F. Oberhettinger, F. G. Tricomi, Higher transcendental functions. Vol. III, McGraw-Hill Book Company, Inc., New York-Toronto-London, (1955). 2.1, 2.1

[15] R. Garraa, R. Garrappa, The Prabhakar or three parameter Mittag-Leffler function: theory and application, Commun. Nonlinear Sci. Numer. Simul., 56 (2018), 314-329. 1

[16] R. Gorenflo, A. A. Kilbas, F. Mainardi, S. V. Rogosin, Mittag-Leffler functions, Related Topics and Applications, Springer, Heidelberg, (2014). 1, 1.2, 2, 2.1, 2.1

[17] R. Goyal, Bicomplex polygamma function, Tokyo J. Math., 30 (2007), 523-530. 1

[18] S. P. Goyal, R. Goyal, On bicomplex Hurwitz zeta function, South East Asian J. Math. Math. Sci., 4 (2006), 59-66. 1

[19] S. P. Goyal, T. Mathur, R. Goyal, Bicomplex Gamma And Beta Function, J. Rajasthan Acad. Phys. Sci., 5 (2006), 131-142. 1, 1.1, 1.1

[20] H. J. Haubold, A. M. Mathai, R. K. Saxena, Mittag-Leffler functions and their applications, J. Appl. Math., 2011 (2011), 51 pages. 1

[21] J. Hucks, Hyperbolic complex structures in physics, J. Math. Phys., 34 (1993), 5986-6008. 1

[22] H. Irmak, P. Agarwal, Some comprehensive inequalities consisting of Mittag-Leffler type functions in the complex plane, Math. Model. Nat. Phenom., 12 (2017), 65-71. 1

[23] S. Jain, P. Agarwal, A. Kilicman, Pathway fractional integral operator associated with 3m-parametric Mittag-Leffler functions, Int. J. Appl. Comput. Math., 4 (2018), 7 pages. 1

[24] R. Kumar, R. Kumar, D. Rochon, The Fundamental Theorems in the framework of Bicomplex Topological Modules, arXiv, (2011), 14 pages. 1

[25] R. Kumar, K. Singh, Bicomplex linear operators on bicomplex Hilbert spaces and Littlewood's subordination theorem, Adv. Appl. Clifford Algebr., 25 (2015), 591-610.

[26] R. Kumar, K. Singh, H. Saini, S. Kumar, Bicomplex weighted Hardy spaces and bicomplex C*-algebras, Adv. Appl. Clifford Algebr., 26 (2016), 217-235.

[27] R. G. Lavoie, L. Marchildon, D. Rochon, The bicomplex quantum harmonic oscillator, Nuovo Cimento Soc. Ital. Fis. B, 125 (2010), 1173-1192.

[28] R. G. Lavoie, L. Marchildon, D. Rochon, Infinite-Dimensional Bicomplex Hilbert Spaces, Ann. Funct. Anal., 1 (2010), 75-91.

[29] R. G. Lavoie, L. Marchildon, D. Rochon, Finite-Dimensional Bicomplex Hilbert Spaces, Adv. Appl. Clifford Algebr., 21 (2011), 561-581. 1

[30] M. E. Luna-Elizarrarás, C. O. Perez-Regalado, M. Shapiro, On linear functionals and Hahn-Banach theorems for hyperbolic and bicomplex modules, Adv. Appl. Clifford Algebr., 24 (2014), 1105-1129. 1.5

[31] M. E. Luna-Elizarrarás, M. Shapiro, D. C. Struppa, A. Vajiac, Bicomplex Numbers and their Elementary Functions, Cubo, 14 (2012), 61-80. 1

[32] D. Alpay, M. E. Luna-Elizarrarás, M. Shapiro Daniele, C. Struppa, Basics of Functional Analysis with bicomplex scalars, and bicomplex Schur analysis, Springer International Publishing, New York, (2014). 1.4

[33] G. Mittag-Leffler, Sur la representation analytiqie d'une fonction monogene (cinquieme note), Acta Math., 29 (1905), 101-181. 1.2

[34] G. B. Price, An Introduction to Multicomplex Spaces and Functions, Marcel Dekker Inc. New York, (1991). 1.1, 1.1

[35] J. D. Riley, Contributions to the theory of functions of a bicomplex variable, Tohoku Math. J., 5 (1953), 132-165. 1.4, 1.1, 2

[36] F. Ringleb, Beiträge zur Funktionentheorie in hyperkomplexen Systemen I., Rend. Circ. Mat. Palermo, 57 (1933), $311-$ 340. 1.1, 1.7

[37] D. Rochon, A Bicomplex Riemann Zeta Function, Tokyo J. Math., 27 (2004), 357-369. 1, 1.1

[38] D. Rochon, M. Shapiro, On algebraic properties of bicomplex and hyperbolic numbers, An. Univ. Oradea Fasc. Mat., 11 (2004), 71-110. 1, 1.1, 1.4, 2.7

[39] D. Rochon, S. Tremblay, Bicomplex quantum mechanics. I. The generalized Schrödinger equation, Adv. Appl. Clifford Algebr., 14 (2004), 231-248. 1.1

[40] D. Rochon, S. Tremblay, Bicomplex quantum mechanics. II. The Hilbert space, Adv. Appl. Clifford Algebr., 16 (2006), 135-157. 1

[41] S. Rönn, Bicomplex algebra and function theory, arXiv, (2001), 71 pages. 1, 1.1

[42] C. Segre, Le rappresentazioni reale delle forme complessee Gli Enti Iperalgebrici, Math. Ann., 40 (1892), 413-467. 1, 1.1

[43] M. F. Yassen, A. A. Attiya, P. Agarwal, Subordination and Superordination properties for certain family of analytic functions associated with Mittag-Leffler function, Symmetry, 12 (2020), 20 pages. 1 